\title{
Estimation of Rural-Urban Expenditure and Elasticities of Food Items in Zambia: Evidence from Living Conditions Monitoring Survey
}

\author{
Musaka Mulanga Chikobola, Abdi-Khalil Edriss \\ Department of Agricultural and Applied Economics, Lilongwe University of Agriculture and Natural Resources, \\ Lilongwe, Malawi \\ Email: mulangamusaka@yahoo.ca, abdikhalil@yahoo.com
}

Received 20 April 2016; accepted 13 May 2016; published 16 May 2016

Copyright (C) 2016 by authors and Scientific Research Publishing Inc. This work is licensed under the Creative Commons Attribution International License (CC BY). http://creativecommons.org/licenses/by/4.0/

\section{Abstract}

The study uses double logarithmic analysis to determine the household food demand pattern in urban and rural regions of Zambia for various stratums. The study utilized secondary data for the 2010 Living Conditions Monitoring Survey (LCMS) from Central Statistical Office (CSO), Government of Zambia. The study used a two-stage cluster sampling procedure to collect the data. Cross sectional data for 19,137 households were used in this analysis. The household size and income elasticities are estimated to explain the food consumption trends in Zambia. The results of the study indicate that all the incomes and some household size elasticities positively influenced food expenditure. The food income elasticity is the highest $(0.6313)$ for the non-agricultural stratum in rural areas and the same is the lowest $(0.2276)$ for high cost stratum in urban areas. Similarly, household size elasticity is the highest $(0.1174)$ for the high cost stratum in urban areas and the same is the lowest $(0.0647)$ for low cost stratum in urban areas. The elasticities tend to be higher in rural areas than urban ones. These differences in consumption patterns between rural and urban areas, and among stratums imply that change in consumption patterns in Zambia will be affected not only by aggregate income growth but also by changes in the distribution of that income to foster food security in the households.

\section{Keywords}

Urban-Rural, Engel Curves, Expenditure and Household Size Elasticities, Zambia 


\section{Introduction}

Zambia is a developing country and falls in the medium Human Development Index (HDI) because it is ranked 141th out of 187 countries [1]. Over $60 \%$ of Zambia's 13 million population lives in rural areas and draws its livelihoods mainly from smallholder production systems centered on agriculture. However, more than half (64\%) of the total population lives below the international poverty line of US $\$ 1.25$ a day [1] [2]. Despite the ease of lifestyles, due to the well modified and modernized system of the availability of goods and services, the conditions of most of the people are still miserable and they are in worry to fulfill even their basic consumption needs. Though over 13 million families are regarded as growing middle class, a large portion of the country's population is still poor. The standard of living in Zambia is differentiated and varies among different social classes. Poverty has also continued to be more of a rural than an urban phenomenon. The level of rural poverty is more than twice that obtaining in urban areas i.e. $80 \%$ of the rural population living in poverty compared to $28 \%$ in urban areas [2].

Household consumption expenditure plays a vital role in the economy in several ways. It is closely linked to household poverty, wellbeing and living standards. Household wellbeing and living standards are judged by the quality and quantity of goods and services that the household is able to access. Household consumption expenditure also constitutes a sizeable proportion of Household Final Consumption Expenditure (HFCE) in the national accounts. HFCE is one of the key indicators used to measure the health and vitality of an economy as well as that of individual households. It is the market value of all goods and services purchased by households. It significantly affects aggregate demand, income and employment in an economy. In Zambia, HFCE is the largest component of Gross Domestic Product (GDP) by type of expenditure, accounting for nearly 60\% of total GDP. Moreover, household consumption expenditure serves as a useful proxy for household income, which in many cases tends to be under-reported by most households.

The progress and development of any economy are related with the consumption in a way that it measures the welfare of the people who are making expenditure on the purchase of various consumption heads. Then again, it is helpful in the extension of business activities because the entire investment set up is dependent upon the consumption pattern in the country [3]. Due to the importance of consumption in the economic theory, researchers have carried out research on household deeds in Zambia and in other countries using different types of data and econometric techniques.

In a recent contribution, Sichilima et al. [4] employed a double-hurdle model to identify factors that affect household decisions on the level of beef expenditure among Lusaka residents in Zambia. Data for 2585 households from the 2010 Living Conditions Monitoring Survey (LCMS) were used. Results showed that rural households make expenditure decisions that are significantly different from urban households. The results also reveal that factors such as income, size of the household, price of beef, price of chicken and education level of the household head are important determinants that influence both the decision to purchase beef and the level of beef expenditure.

Another study by Chongela et al. [5] employed the Almost Ideal Demand System (AIDS) to estimate the consumer demand system of agri-food consumed by the households in Tanzania. Secondary from Household Budget Survey (HBS) conducted in 2007 by the National Bureau of Statistics (NBS) of the United Republic of Tanzania was used. Empirical results revealed that households' food budget share was $60 \%$ on average per month. The own price elasticity of demand for aggregated agri-food was inelastic (0.86). Income elasticity of demand for aggregated agri-food was 0.96. Results suggested government intervention in terms of agri-food price stabilization policies and programmes as well as income support policies.

In Egypt, Dawoud [6] analysed the changes in food expenditure patterns over time with special emphasis on the differences between urban and rural sectors. Data used in the study were obtained from the Household, Income, Expenditure, and Consumption Survey (HIECS) conducted by the Central Agency for Public Mobilisation and Statistics (CAPMAS) of Egypt for five survey periods from 1990/1991 to 2009/2010. The Engel curves for food groups were estimated by using double-log function type. The results showed statistically significant variations between the urban and rural expenditure elasticity of most food commodities and that elasticities tend to be higher in rural areas than urban ones. The expenditure elasticities of food groups are lower at high-income groups than low-income ones. The results provided the guideline for future policy implication in respect of the demand management and food consumption in Egypt.

This paper aims to estimate econometrically the food expenditure patterns in Zambia. Special emphasis is on 
the difference between urban and rural areas, as well as at different household stratums, since there is paucity of empirical literature. The specific objectives are: 1) to measure the economies of scale effect in household consumption by including the household size as an independent variable in Engel curve equation; 2) to estimate expenditure and household size elasticities of food demand and suggest some implications for policy.

The estimation of food consumption pattern reveals a clear representation of living standard and the economic growth of the country. Therefore, policy formulation and poverty reduction have a special need for household expenditure data analysis. The results of this study could provide guidelines for future policy implication in respect of the management of the demand and consumption of food commodities in the country.

\section{Methodology}

\subsection{Data}

The data utilized in this study was from the Government of Zambia's 2010 Living Conditions Monitoring Survey (LCMS) conducted by the Central Statistical Office (CSO). The nationally representative survey had a total sample size of 20,000 households distributed across 1000 Standard Enumeration Areas (SEAs). A two-stage cluster sampling procedure was used in which the first stage involved selection of Primary Sampling Units (PSUs) or SEAs using Probability Proportional to Estimated Size (PPES) from a national sampling frame developed during the 2000 Census of population and housing. The second stage then involved using systematic sampling to select the sample households in the SEAs that had been selected in the first stage. The sampling frame for the second stage was developed through a comprehensive listing exercise. During the 2000 census, the country was administratively demarcated into 9 provinces, which were further sub-divided into 72 districts. The districts were further subdivided into a total of 150 constituencies, which were in turn divided into wards. For the purposes of facilitating CSO surveys, wards were further divided into Census Supervisory Areas (CSA), which were further subdivided into SEAs. SEAs constitute the PSUs. The allocation of the sample points to rural and urban strata was done in such a way that it was proportional to their sizes in each district. Data for 19,137 households was used in this analysis.

\subsection{Theoretical Foundation}

Household demand is a combination of two important words household and demand. Household refers to a single person or a group of persons who normally live and eat together [2], while the demand is the quantities of goods and services that the consumers are willing and able to buy from the market at various prices. Consumption of various goods and services is the reflection of household expenditure pattern which are largely influenced by household composition, needs, taste and financial means. The utility concept is used to define the level of satisfaction or welfare that comes from a specific allocation of income among different products. In economic theory, the relationship between income level and the quantity purchased is interpreted by income consumption curves. German statistician Ernst Engel [7] had established this approach firstly in the 19th century. Since then the curve that shows the influence of the changes in the consumer income on the quantity demanded is called Engel curve. The household expenditure behaviour can be analyzed using Engel curves [8]. Engel curve shows how the preferences between goods change when there is an increase in the household income while the prices of the goods are fixed. Households primarily tend to satisfy their household's basic needs and as the level of welfare increases, the share of expenditure on necessities such as food decreases.

The relationship between demand and total expenditure can be used to derive expenditure elasticities of demand for goods. These elasticities represent the percentage of change in the quantity demanded as a response to the percentage change in the income level. According to Engel curve, the commodities are classified into two categories as necessities and luxuries. If the income elasticity of demand for certain goods is less than one, such goods are necessities and if it is larger than one, it would be luxury goods. Engel (Expenditure) elasticities are a powerful research tool in the household analysis. In economics the variation from proportionality of one variable with respect to another variable is measured by elasticity. Thus, if expenditure on a certain item is proportional to income or total expenditure then the income elasticity of demand, better known as Engel elasticity is unity. On the other hand, if expenditure on a certain item rises more proportionately relative to income, the Engel elasticity is greater than one. Similarly, if expenditure on an item rises less than proportionately relative to income, the Engel elasticity will be less than one. This concept is helpful in categorizing the commodities into a necessi- 
ty, normal and luxury if it has Engel elasticity less than one, equal to one and greater than unity, respectively.

The most available data of Zambian Household Surveys are completed in a short time-span, and thus the prices faced by all households can be regarded as constant. This allows focus on responses of household demand to variations in income or total expenditure. Therefore, this study employed the specification of the Engel model, which uses only expenditure data. There are many functional forms that are used to estimate Engel curves. In this study, a double logarithmic functional form is used to estimate expenditure elasticities because of its simplicity, easy estimation and interpretation [9]. Also, expenditure coefficient is the coefficient of elasticity and there is no need of calculation.

In estimation of Engel curves, total expenditure is commonly used as a proxy of income for two reasons [10] [11]: First, total household expenditure tends to be more accurately reported, is easier to measure than total household income, and is measured with less error of measurement particularity in developing countries. Second; income may be subject to transitory fluctuations since savings allow smoothing of expenditure over time. Thus, the total expenditure elasticities are calculated instead of income elasticities. A functional form that is termed as the constant elasticity, log-log or double log specification which is undertaken in this study has been widely used for demand analysis [3] [7] [12]. Though such a system is not consistent with the budget constraint and the theoretical restrictions on systems of demand equations but this is a frequently used specification of a system of demand equations and at best can be treated as a local approximation to the true system of demand equations [13].

\subsection{Econometric Construct}

A double-log specification has proven the most appropriate way of estimating the expenditure elasticity of demand and it generates more realistic expenditure elasticities [14]. Consider a complete system of demand equations for $\mathrm{n}$ goods consists of the $\mathrm{n}$ demand equations:

$$
X_{j}=X_{j}\left(P_{1}, P_{2}, \cdots, P_{n}, I, \mu_{j}\right), \quad j=1,2,3, \cdots, n
$$

where $X_{j}$ is the demand for good $j$ by a single household or a group of households, $P_{j}$ is the price of good $j, I$ is income which is the same as the expenditure on the $n$ goods and $\mu_{j}$ is the stochastic term in the $j$ th demand equation. In order to estimate the system (1) it is necessary to specify a particular functional form for the estimation of general relationship. Thus the $\mathrm{n}$ demand functions in (1) are specified as:

$$
X_{j}=A_{j} P_{1}^{\eta j 1} P_{2}^{\eta j 2} \cdots P_{n}^{\eta j n} I_{n}^{\eta j 1} \mathrm{e}^{\mu j}
$$

The linearization of (2) by taking logarithms leads to the log-log specification of the form.

$$
\ln X_{j}=\alpha_{j}+\eta_{j 1} \ln P_{1}+\eta_{j 2} \ln P_{2}+\cdots+\eta_{j n} \ln P_{n}+\eta_{j} \ln I+\mu_{j}
$$

where $\alpha_{j}=1 n A_{j}$ considering the price effect constant, the $\eta_{j}$ are the income elasticities of demand that can be computed as:

$$
\eta_{j}=\frac{\Delta \ln X_{j}}{\Delta \ln I}=\frac{\Delta X_{j}}{\Delta I} * \frac{I}{X_{j}}
$$

Present study uses a double logarithmic functional form to estimate demand pattern because of its certain advantages. It is simple as its estimation and interpretation is quite easy. It is most appropriate to estimate the demand pattern of grouped commodities [15]. The income or expenditure coefficient is also the coefficient of elasticity so there is no need of further calculation. It is an easy technique to determine the most efficient effect of household size [7]. Thus, by incorporating total expenditure and household size as an independent variables in Engel curve equation and taking the natural log gives:

$$
\ln F_{i j}=\alpha_{i j}+\beta_{i j} \ln E_{j}+\gamma_{i j} \ln H S_{j}+\mu_{i j}
$$

where $F_{i}$ is food expenditure by $i$ th household, $E_{j}$ is total expenditure by $j$ th household, $H S_{j}$ is household size and $\mu_{i}$ is the stochastic error term. The food expenditures are the sum of expenditures by each household on the following commodity groups: cereals, roots and tubers, pulses and legumes, vegetables, fruits, fish, meat and poultry, dairy products and egg and the non-alcoholic beverages, including own produce consumed. 


\section{Results and Discussion}

The study used the double logarithmic regression analysis to compute the household food demand pattern by computing its relationship with household total expenditures and household size. The estimation was done for both rural and urban areas of Zambia separately by dividing the households into seven stratum. Ordinary Least Square (OLS) regression technique was employed. Estimation of double log regression (Equation (5)) gives estimates of food expenditure and household size elasticities.

\subsection{Diagnostic Tests}

After fitting the double logarithmic model, the variables were checked for the existence of multicollinearity and normality. The mean VIF of 1.03 was found and this showed that there was no serious problem of multicollinearity in the model since the value of VIF found is less than 10 . The normality test was also done using the kernel density plot of residuals. The kernel density plot provided a fairly smooth curve that closely matched the normal curve (see Figure 1). Hence the normality assumption was not violated.

\subsection{Expenditure and Household Size Elasticities Estimates}

The expenditure and household size elasticities are estimated to explain the food consumption trends in Zambia. Here, the total expenditure is used as a proxy of income. The results of the expenditure and household size elasticities for urban and rural areas are shown in Table 1. With respect to total expenditure, the results show that the elasticities of all the consumption items are positive. All the total expenditure (income) elasticities for both the urban and rural regions are significant at $1 \%$ level, implying that all food items include in the analysis are necessities in the whole computation.

The results also show that elasticities tend to be higher in rural areas than urban ones. The differences in consumption patterns between rural and urban areas, and among stratums imply that change in consumption patterns in Zambia will be affected not only by aggregate income growth but also by changes in the distribution of that income. The previous studies in Egypt and Tanzania [5] [6] found similar results. Table 1 shows that food income elasticity is the highest (0.6313) for the non-agricultural stratum in rural areas and then same is the lowest $(0.2276)$ for high cost stratum in urban areas. The results implies that as incomes get higher, a smaller percentage of income goes to consumption and consumption thus increases at a decreasing rate. For a $1 \%$ increase in income there will be a $63.13 \%$ and $22.76 \%$ increase in the monthly food expenditure for non-agricultural and high cost stratums respectively. From the estimation results, a one percentage point fall in income would reduce food consumption by 0.4491 percentage points overall. This is a sign of poverty since very little is saved for investment especially from rural stratum households (Table 1).

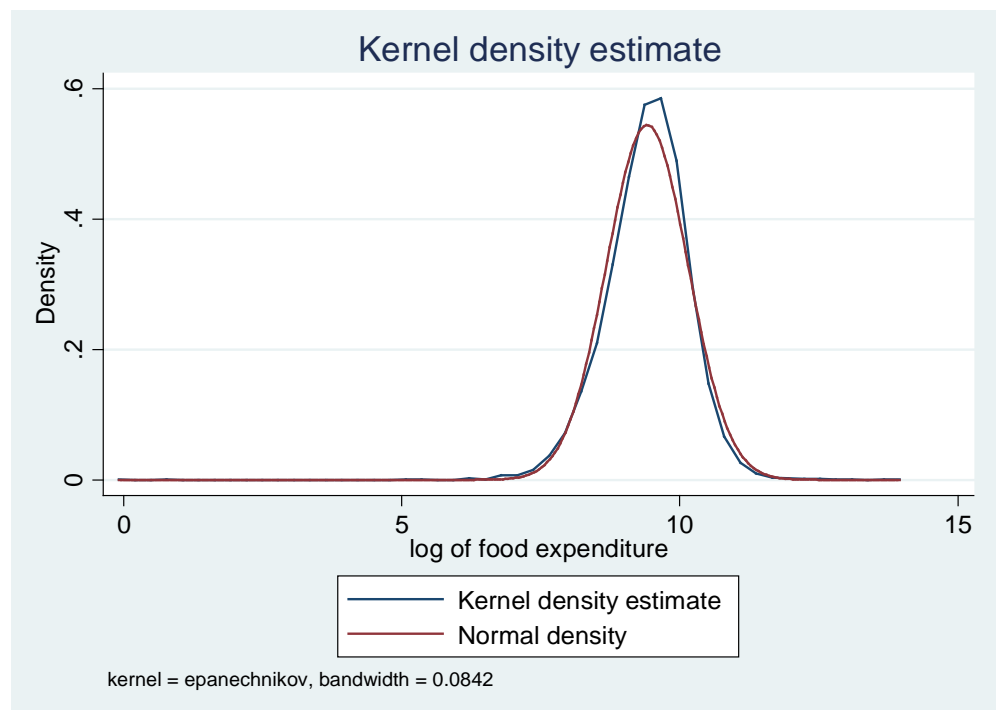

Figure 1. Test for normality. 
Table 1. Expenditure and household size elasticities.

\begin{tabular}{|c|c|c|c|c|c|c|}
\hline Household stratum & Constant & $\mathrm{Ej}$ & HSj & R-squared & F-stat & df \\
\hline \multicolumn{7}{|l|}{ Rural stratum } \\
\hline \multirow[t]{2}{*}{ Small scale } & $3.2689 a$ & 0.6130a & $0.0153^{\mathrm{NS}}$ & 0.5961 & 4437.26a & 6014 \\
\hline & $(-)$ & $(0.7697)$ & $(0.0104)$ & & & \\
\hline \multirow[t]{2}{*}{ Medium scale } & 5.4199a & $0.3828 a$ & $0.0737 \mathrm{c}$ & 0.3750 & $278.97 a$ & 930 \\
\hline & $(-)$ & $(0.6007)$ & $(0.0280)$ & & & \\
\hline \multirow[t]{2}{*}{ Large scale } & $6.1156 a$ & $0.3504 a$ & $0.0052^{\mathrm{NS}}$ & 0.4198 & $19.90 \mathrm{a}$ & 55 \\
\hline & $(-)$ & $(0.6450)$ & $(0.0253)$ & & & \\
\hline \multirow[t]{2}{*}{ Non-agricultural } & 3.1758a & 0.6313a & $0.0209^{\mathrm{NS}}$ & 0.6586 & 1171.86a & 1215 \\
\hline & $(-)$ & $(0.7402)$ & $(0.0725)$ & & & \\
\hline \multicolumn{7}{|l|}{ Urban stratum } \\
\hline \multirow[t]{2}{*}{ Low cost } & $5.4368 a$ & $0.3384 a$ & $0.0647 a$ & 0.4319 & 2639.58a & 6945 \\
\hline & $(-)$ & $(0.6397)$ & $(0.0621)$ & & & \\
\hline \multirow[t]{2}{*}{ Medium cost } & 6.8003a & $0.2627 a$ & 0.1038 a & 0.3351 & $571.29 a$ & 2267 \\
\hline & $(-)$ & $(0.5436)$ & $(0.1073)$ & & & \\
\hline \multirow[t]{2}{*}{ High cost } & $7.2346 a$ & $0.2276 a$ & $0.1174 a$ & 0.3404 & 436.06a & 1690 \\
\hline & $(-)$ & $(0.5279)$ & $(0.1414)$ & & & \\
\hline \multirow[t]{2}{*}{ Overall } & 4.8496a & $0.4491 \mathrm{a}$ & $0.0385 a$ & 0.5230 & 10488.98a & 19134 \\
\hline & $(-)$ & $(0.7161)$ & $(0.0312)$ & & & \\
\hline
\end{tabular}

Note: a and c stands for statistical significance tests at $1 \%$ and $10 \%$ levels respectively; NS = not statistically significant; Figures in parentheses are beta coefficients; $\mathrm{Ej}$ = total expenditure (proxy for the household income), $\mathrm{HSj}$ = household size.

The household size is used also as an explanatory variable in order to investigate the economies of scale effect in the food demand pattern for various stratum. The computed household size elasticities at national level are given in Table 1. The household size elasticities were significant at $10 \%$ level for the medium scale stratum in the rural areas; and were significant at $1 \%$ level for all the stratums in the urban areas. Results also show that household size elasticity is the highest (0.1174) for the high cost stratum in urban areas and the same is the lowest (0.0647) for low cost stratum in urban areas. Thus for a $1 \%$ increase in household size there will be a $11.74 \%$ and $6.47 \%$ increase in the monthly food expenditure for high cost and low cost stratums respectively. Most of the elasticity coefficients exhibit quite little cyclical fluctuations among various stratum.

It is notable in Table 1 that the total expenditure elasticity for food is positive and less than unity, thus confirming the Engel's law. The results further shows that the elasticity coefficient of the size of the household with respect to all expenditure items are less than unity. Similar results were obtained by Rehman et al. [16] in Pakistan and Sichilima et al. [4] in Zambia. In general, the regression results demonstrate that the total household expenditure (used as a proxy of income) and size of the household is an important determinant of expenditures on food. Moreover, the overall sample regression results have also demonstrated that income and household size are significant determinant of the expenditure on the commodity groups investigated. The beta statistic reveals, however, that income has a higher explanatory power than the household size. This result underscores income as the single most important variable which influenced the household expenditures on the commodity groups analyzed in this study.

It is worth noting that, the unadjusted coefficient of determination $\left(R^{2}\right)$ is generally very low, but specifically relatively large (0.5230) in the function estimated for the food items. This implies the regressands explained only about $50 \%$ of the variation in the expenditures on food. On the other hand, the F-statistics are statistically significant in all of functions fitted for different stratums. The significance of the F-statistics obtained implies that 
the explanatory variables explain variation in the dependent variables of the functions estimated.

\section{Conclusions}

This paper analyzed the applicability of the Engel's law in Zambia by using a sample of 19,137 households covered by the Living Conditions Monitoring Survey of 2010. The legitimacy of Engel's law was verified because the proportion of food consumption is lower as compared to income. The household size analysis confirms the existence of economies of scale for food consumption among different strata classifications. Urban stratum households depict higher size elasticities in overall compared to rural households belonging to rural stratums.

The estimation results of this study have some implications for policy. Of particular interest is the income (total expenditure) and household size elasticities. The overall sample income elasticity with respect to food first, implies a prevalence of very low standard of living in the sampled areas. These aspects underscore the importance of policies aimed at increasing incomes and fostering food security in the households. Anti-inflationary policy measures such as better inputs which would increase the supply of food could be pursued. The income augmenting policies need to be complemented with a sensitization of family planning practices. This is important because the empirical results demonstrate a positive and statistically significant effect of the household size on expenditures on food.

\section{Suggestions for Further Research}

Further work is required to capture the effects of gender, locational factors, dependency ratio etc., on the consumption pattern of the households. These have not been modelled here because of some inadequacies inherent in the data collected. Additionally, use of the results presented in this paper for policy and planning purposes is undermined by changes resulting from macro-economic stabilization programmes put in practice since 2010 when the data used were collected. Undoubtedly, these programmes may have influenced the consumption pattern in Zambia, nevertheless to what extent is a question which remains to be established empirically.

\section{References}

[1] UNDP (United Nations Development Programme) (2014) Human Development Report 2014: Sustaining Human Progress; Reducing Vulnerability and Building Resilience. New York.

[2] CSO (Central Statistical Office) (2012) Living Conditions Monitoring Survey Report (2006 and 2010). Lusaka.

[3] Sher, F., Ahmad, N. and Safdar, S. (2012) Income and Economies of Scale Effect on Household Food Demand Pattern in Pakistan Using PSLM Data. Academic Research International, 3, 50-56.

[4] Sichilima, T., Mapemba, L. and Tembo, G. (2015) What Determines Expenditure Allocation to Beef among Lusaka Residents in Zambia? Evidence from Household Survey. Modern Economy, 6, 411-422. http://dx.doi.org/10.4236/me.2015.63039

[5] Chongela, J., Nandala, V. and Korabandi, S. (2014) Consumer Demand System of Agri-Food in Tanzania. Journal of Development and Agricultural Economics, 6, 42-48. http://dx.doi.org/10.5897/JDAE2013.0475

[6] Dawoud, S.D.Z. (2014) Econometric Analysis of the Changes in Food Consumption Expenditure Patterns in Egypt. Journal of Development and Agricultural Economics, 6, 1-11. http://dx.doi.org/10.5897/JDAE2013.0511

[7] Houthakker, H.S. (1957) An International Comparison of Household Expenditure Patterns, Commemorating the Centenary of Engle's Law. Econometrica, 25, 532-551. http://dx.doi.org/10.2307/1905382

[8] Sadoulet, E. and Janury, A. (1995) Quantitative Development Policy Analysis. The John Hopkins University Press, Baltimore.

[9] Ahmed, N., Safdar, S. and Sher, F. (2012) Analysis of Income and Economies of Scale Effect on Household Demand in Pakistan Using PSLM Data. Interdisciplinary Journal of Contemporary Research in Business, 3, 117-124.

[10] Deaton, A. (1997) The Analysis of Household Surveys: A Micro Econometric Approach to Development Policy. The John Hopkins University Press, Baltimore. http://dx.doi.org/10.1596/0-8018-5254-4

[11] Tansel, A. (2002) Determinants of School Attainment of Boys and Girls in Turkey: Individual, Household and Community Factors. Economic Education Review, 21, 455-470. http://dx.doi.org/10.1016/S0272-7757(01)00028-0

[12] Burney, N.A. and Khan, A.H. (1991) Household Consumption Patterns in Pakistan: An Urban Rural Comparison Using Micro Data. The Pakistan Development Review, 30, 145-171.

[13] Griliches, Z. and Intriligator, M.D. (1983) Economic and Econometric Models. North-Holland Publishing Company, 
Holland.

[14] Babar, A. and Shahnawaz, M. (2010) Household Consumption Patterns in Pakistan: A Rural Urban Analysis. Forman Journal of Economic Studies, 6, 1-25.

[15] Islam, M. and Siwar, C. (2005) Impact of the Financial Crisis on Expenditure Patterns in Malaysia: Special Reference on Low-Income Households. Journal of Economic Research, 10, 145-173.

[16] Rehman, A., Wang, J. and Zhang, R.Q. (2014) Estimation of Urban-Rural Expenditure and Household Size Elasticities of Food Items in Pakistan: Evidence from PSLM Survey. Asian Economic and Financial Review, 4, 183-190. 